\title{
LA FORMACIÓN DUAL Y SU FUNDAMENTACIÓN CURRICULAR
}

\author{
Isabel Araya Muñoz \\ Estudiante de la Maestría en Planificación Curricular \\ Universidad de Costa Rica \\ San José, Costa Rica
}

Recibido: 27-XI-2007 • Aceptado 11-III-2008 • Corregido 15-IV-2008

\begin{abstract}
Resumen: La educación dual es una modalidad de enseñanza y de aprendizaje que se realiza en dos lugares distintos; la institución educativa y la empresa, que se complementan mediante actividades coordinadas. El principio fundamental de este modelo pedagógico corresponde a la relación educación-trabajo en la formación profesional, que se cimienta en los enfoques tecnológico y humanista y debe ser abordado desde perspectivas filosóficas para determinar el tipo de ciudadano que se desea formar, sus valores universales como individuo y a partir de su convivencia con otros, desde el fundamento epistemológico define el saber y las diversas modalidades de conocimiento, su fundamento psicopedagógico comprende un proceso de enseñanza y de aprendizaje que interrelaciona la psicología y la pedagogía para orientar esa actividad en dos lugares distintos, la escuela y la empresa, mediante un enlace cooperativo, el fundamento socioeconómico que establece la visión de formación del recurso humano para la productividad y que se apega al desarrollo económico y social del individuo y de la sociedad en la que está inmersa.
\end{abstract}

Palabras clave: Educación dual, formación dual, modalidad pedagógica, modelo pedagógico, sistema alemán, fundamentos curriculares, educación-empresa, educación-experiencia, educación-trabajo.

\section{Introducción}

El propósito de este artículo es resaltar la importancia del principio de la relación educación-trabajo en la formación profesional como fundamento de la formación dual dentro de la concepción curricular humanista y tecnológica y desde las perspectivas filosóficas, epistemológicas, psicopedagógicas y socioeconómicas en las que se establece un proyecto curricular en esa modalidad de enseñanza y de aprendizaje.

Esta modalidad educativa de reciente aplicación en Costa Rica es una propuesta académica innovadora que además busca el aprovechamiento de los recursos empresariales, los de la institución educativa y el aporte del estudiante, partícipes todos en una tarea coordinada, en las que hay un aprovechamiento de la interacción de los sujetos, a partir de un enlace cooperativo.

Este proceso educativo, a diferencia del tradicional en el que el docente asume una posición de poder, pasa a ser una actividad dinámica. Así, tanto el participante como el facilitador deben aplicar principios de autorresponsabilidad, de análisis crítico y de creatividad en la concepción de cada etapa de ese proceso y con ello promueven 


\begin{abstract}
The dual education is a modality of education and of learning that is realized in two different places, the educational institution and the company that, by means of coordinated activities, complement each other. The fundamental principle of this pedagogic model corresponds to the relationship education-work in the vocational training, founded on the technological and humanist approaches and must be viewed from the philosophical perspectives to determine the type of citizen who is wanted to be formed, his universal values as individual and from his living together with others, from the epistemological foundation it defines the knowledge and its diverse modalities, its psycho pedagogic foundation understands a process of education and of learning that interrelates the psychology and the pedagogy to guide this activity in two different places, the school and the company, by means of a cooperative linkage, the socioeconomic foundation that establishes the vision of formation of the human resource for productivity and that is attached to the economic and social development of the individual and of the society in which it is immersed.
\end{abstract}

Key words: Dual education, dual formation, pedagogic modality, pedagogic model, german system, curricular foundations, education-company, education -experience, education-work. la formación de profesionales capaces de aplicar criterios y procedimientos con ética y madurez.

El propósito principal de la formación dual está orientado a un proceso educativo integral, a través de una alianza estratégica entre la empresa y la academia. En este proceso, el estudiante alcanza un nivel de desarrollo en un puesto de trabajo que le permitirá competir como un profesional altamente calificado por sus cualidades humanas, intelectuales, prácticas y actitudinales. Por su parte, la empresa recibe un aporte de conocimiento, a partir del aporte del alumno, así también la institución educativa actualiza y enriquece su quehacer académico con base en las necesidades reales de formación, que sistematiza a partir de la experiencia del estudiante.

La dualidad, academia y empresa, ubica el principio fundamental de este hecho educativo admitiendo a la segunda como una nueva escuela, donde el estudiante aprende por medio de la práctica en situaciones o problemas reales de un puesto de trabajo y mediante la aplicación de principios teóricos logra la transformación de la realidad.

La formación dual (Vega, 2005), la define como una modalidad de formación profesional, y por ende educativa, que realiza su proceso de enseñanza-aprendizaje en dos lugares distintos, en una institución educativa donde realiza actividades teóricas-prácticas y en una organización donde ejecuta actividades didáctico-productivas que se complementan y se alternan.

Ese proceso implica la integración de diferentes componentes, tales como conocimientos teóricos, trabajo de aula, laboratorio y la utilización de recursos existentes en el medio laboral, además del aprovechamiento de la capacidad instalada de la empresa. El estudiante recibe el apoyo, instrucción y orientación de un guía o tutor en la empresa, quien es un funcionario experimentado y con conocimientos en la especialidad. El tutor, mediante la observación directa, se encarga de darle 
seguimiento al trabajo práctico que realiza el estudiante, para lograr la potenciación de sus capacidades, mediante la adquisición de nuevas competencias, y a través de la coordinación de un profesor acompañante de parte de la institución educativa, quien da seguimiento metodológico a ese proceso de aprendizaje.

La importancia que reviste esta modalidad de aprendizaje consiste en cuatro elementos curriculares fundamentales en un proceso dinámico, participativo, que reconoce al estudiante en un aprendizaje realidad en el que se adapta desde muy joven al mundo laboral aprendiendo. Los elementos son: a) aprendizaje significativo del estudiante, quien no solo aprende contenidos teóricos, sino que adquiere competencias que a veces los libros no contemplan; b) el aprovechamiento y la aplicación de herramientas tecnológicas y procedimentales actualizadas que no dispone la institución educativa pública y que la empresa actual posee; c) insumo vital para la institución educativa en la actualización de los planes de estudio según las necesidades de formación, debido a los avances tecnológicos tan acelerados que exigen los estándares de eficiencia y competitividad en las empresas y d) el aporte de conocimiento de la institución educativa a la empresa por medio de la interacción estudiantes, profesores acompañantes y tutores.

Considerando esos elementos curriculares la relación educación-trabajo como principio de la formación dual se fundamenta en una concepción curricular humanista y tecnológica-tyleriana, a partir de perspectivas filosóficas, epistemológicas, psicopedagógicas y socioeconómicas para establecer un diseño curricular que remite a aspectos propios del proceso de enseñanza y de aprendizaje y a la noción de una serie de conocimientos, habilidades y actitudes con una orientación de la educación para la vida. Esto es posible con un currículo integrado que posee ventajas sobre el tradicional, que se basa en objetivos y a la mera transmisión de conocimientos teóricos y prácticos en situaciones simuladas.

La integración curricular facilita el contacto del estudiante con la realidad productiva en la que adquiere conocimiento, por tanto el enfoque se orienta hacia la educación centrada en el estudiante en torno a la realidad, a la cual se acerca por medio de la práctica de la enseñanza que involucra la vivencia y la contextualización de los contenidos curriculares, la participación práctica y la incorporación de las exigencias del contexto económico, la necesidad de actualización del docente y la orientación hacia la innovación, por tanto toma en cuenta las aptitudes y actitudes de los sujetos, su necesidad de formación, los recursos disponibles y el interés para proveer situaciones que faciliten un aprendizaje significativo.

La estructura de este artículo está conformada por tres apartados, el primero comprende los antecedentes de la formación dual y algunos indicios de sus orígenes como modalidad de enseñanza y de aprendizaje en un lugar distinto al aula tradicional, a partir del involucramiento del aprender haciendo en una situación real de ejecución de tareas, así como la experiencia de Alemania, país al que se le atribuye el surgimiento de esa propuesta pedagógica y que ha sido propulsor de esta modalidad en América Latina, cuyo propósito es darle una mayor participación a la actividad práctica en la enseñanza técnica y profesional con miras a fortalecer la formación del recurso humano.

El segundo apartado describe el principio educación-trabajo en la formación de profesionales. Este principio se constituye en una estrategia ejecutora de la actividad didáctico-productiva hacia la innovación y nuevas posibilidades de desarrollo del capital humano y a los cambios que exige la empresa actual.

En el último apartado, se presentan los fundamentos curriculares que sustentan un programa educativo en la modalidad dual. 


\section{Antecedentes de la Formación Dual}

El valor fundamental de la educación en el desarrollo del ser humano con miras de satisfacer las necesidades individuales y sociales ha sido, durante la historia, preocupación de muchos por darle el mejor aprovechamiento a los recursos y lograr productividad con la mejor calidad. Ante esos requerimientos de formación, cada vez son mayores los espacios emergentes para hacer nuevas ofertas que respondan a los cambios socioeconómicos en términos de profesionales altamente calificados, y la formación dual es una propuesta educativa que se enfatiza en el aprovechamiento del ejercicio práctico, paralelo a la enseñanza escolarizada. Es una propuesta que surge como respuesta a esas necesidades, pero ¿qué le antecede a esa propuesta actual de mejora de la formación del recurso humano? ¿a qué se le atribuye el origen y desarrollo de esta modalidad educativa?

Las raíces de la formación dual, según (Mittmann, 2001), datan de la Edad Media en Europa, originada en el aprender haciendo bajo la supervisión de un maestro, que luego fue normado por los gremios de artesanos en el que el maestro era responsable de la educación del joven, la formación del carácter y la enseñanza de un oficio. El aprendiz no recibía ningún tipo de salario, pero se le brindaba alojamiento, alimentación y ropa, cuando terminaba el aprendizaje se convertía en oficial y recibía el salario correspondiente. (Tünnermann, 1997) también atribuye el aprendizaje de los oficios en esa misma época al relacionar la universitas a los gremios, corporaciones o fraternidades integradas por personas dedicadas a una misma tarea, entre quienes se distinguían los maestros (magisteri) de los aprendices (discipuli).

Esas dos propuestas podrían dar indicios sobre el origen de sistematizar el aprendizaje de oficios y saberes, como lo indica (Neave, 2001) a partir de ésa época se fortalece la estructura del sistema tradicional como institución por medio del studium generale, en la Universidad de Bolonia en 1140 y el aprender en un puesto de trabajo en la empresa. A partir de Bolonia, ya con la aparición del Estado-nación, surge un nuevo principio rector en la educación, la utilidad del conocimiento, con miras a fomentar, el credo y la fe que sustentaban los primeros principios educativos, la relación gobierno-educación priva en la introducción de conocimientos científicos para un estado profesionalizado que requería de personal para el resguardo, el orden público, la salud y la infraestructura.

En la línea de la formación técnica en centros fuera del aula tradicional (Neave, 2001) manifiesta que la iniciativa francesa en este modelo educativo se ilustra mediante la diversificación institucional con la creación en 1773 de la Ecole des Ponts et Chaussées como un establecimiento para enseñar a ingenieros viales y que más tarde generó el modelo de la Ecole Polythecnique para instruir en técnicas militares, eso resalta la necesidad de que las dependencias del Estado tengan sus propios establecimientos para formar a los especialistas fuera de la universidad.

Otro precedente de la formación dual lo atribuye (Rolf, 1997) a las escuelas dominicales, religiosas y comerciales de los siglos XVII y XVIII, en las que los aprendices asistían a clases los domingos o por las noches y a las escuelas de perfeccionamiento profesional en el siglo XIX, que se consideran antecesoras de los actuales centros de educación dual en Alemania y que fueron establecidas formalmente con el Reglamento Comercial e Industrial, decretado en 1869 por la Confederación del Norte de Alemania. Esa fue una oportunidad importante para la formalización del sistema de formación dual vigente.

Tal y como lo indica (Rolf, 1997) la formación dual recibió su nombre actual a raíz de la definición aplicada por la Comisión Alemana de Educación y Cultura en 1964, a partir del Dictamen Pericial sobre la Formación Profesional y la Educación 
Escolar, y de las escuelas de perfeccionamiento profesional en el transcurso del siglo XIX, que se consideran antecesoras de los actuales centros de enseñanza.

Esa tradición ha sido una fuente de inspiración que ha producido trabajadores que dominan plenamente sus conocimientos técnicos y los transmiten, respondiendo a lo que (Dewey, 1976) estableció sobre el conocimiento y su necesidad de aplicación para hacer el enlace entre sujeto-objeto por medio del "conocer-hacer, teoría-práctica" y que se confirma también en las teorías administrativas contemporáneas en la formación del recurso humano, que según (Stoner y Wankel, 1989) en la empresa existe el mentor, persona que en la empresa tiene el papel central de ayudar a otros para el logro de una carrera exitosa.

Queda establecido así que la educación dual se fundamenta en la actividad educativa a partir de la enseñanza en un puesto de trabajo y que en esa capacitación del recurso humano, se establece una relación teoría-práctica, a partir de la integración de conocimientos, por tanto, a través de la historia se concibe la necesidad de llevar la academia a la empresa.

La necesidad de enriquecer el potencial del recurso humano mediante la formación académica y práctica es fundamental para el logro de profesionales altamente calificados. Esas cualidades de la persona formada las establece (Stoner y Wankel, 1989), en tres categorías básicas, destrezas técnicas mediante las cuales se aprende a utilizar procedimientos y técnicas propias de una disciplina, destrezas humanas para el desarrollo de la capacidad para trabajar con otros y las destrezas conceptuales por la capacidad mental de coordinar e integrar conocimientos y relacionarlos con la realidad en un proceso interactivo academia-empresa, base de la educación dual y que favorece la formación de la persona, situación que ha sido inquietud de la educación secundaria, preuniversitaria y universitaria.

Con el fin de evidenciar la aplicación de la actividad educativa como modalidad dual, se reseña la práctica alemana, país fundador y propulsor en algunos países latinoamericanos, cuya experiencia está debidamente establecida desde la perspectiva socioeconómica y psicopedagógica.

El sistema dual en Alemania está conformado por aproximadamente la mitad de los estudiantes del segundo ciclo y tiene como objetivo brindar una formación profesional de calidad y desarrollar en el educando las competencias necesarias para ocupar un puesto de trabajo calificado. La formación dual otorgada por las empresas y por la escuela está descrita en un plan general que comprende la capacitación para el desempeño de aproximadamente 355 ocupaciones, con algunas variaciones entre un estado y otro. Según (Caillods, s.f.), en Alemania, la gesamtschule o escuela polivalente se introdujo en los setentas en forma experimental con fines de desarrollar programas teóricos y prácticos en alternancia por medio del involucramiento de empresas en la formación. Esa cualificación profesional es reconocida en el mercado de trabajo.

La empresa alemana está acostumbrada a la formación dual y asigna importantes recursos para asegurar la inclusión de estudiantes, así como su buen nivel de implementación. En 1998, el 50\% de los estudiantes de 18 años estudiaba en el sistema dual, el 19\%, en escuelas profesionales a tiempo completo y el $31 \%$ en escuelas generales gymnasium o gesammtschule (Caillods, s.f.).

Alemania presenta nuevas tendencias generales en la formación profesional reorientando la profesión y el desarrollo de nuevas competencias producto de la segmentanción del mercado laboral, el incremento de servicios y los nuevos desarrollos tecnológicos y de telecomunicaciones, que no solo exigen nuevas modalidades de calificación, sino que se acompañan de cambios organizacionales. Esos intereses de una calificación profesional superior (Rolf, 1997) los fundamenta en la orientación de las sociedades modernas como Alemania al cambio de las culturas de aprendizaje hacia 
un aprender organizacional y orientado a las experiencias.

El procedimiento para financiar la formación profesional en Alemania, según (Oviedo y Vargas, 2003) es por medio de convenios de cooperación entre los sectores empresariales y el Estado, de esta manera se avanza en la preparación de mano de obra calificada. Indican también que el tema del sistema dual promovido en Alemania se extiende hacia toda la Unión Europea por medio de la Organización Mundial del Comercio.

En Alemania, la Ley de Formación Profesional regula la formación profesional en la medida en que ésta no se desarrolla en las escuelas profesionales, sujeta a las leyes escolares de los estados federales; además, la ley define la formación profesional en la empresa como una tarea pública, que se deja en gran parte en manos de la economía de mercado, tal y como lo afirman (Tippelt y Amorós, 2000).

Alemania es un modelo de desarrollo para esta propuesta educativa y posee una estructura cooperativa entre empresas estatales y privadas que fortalecen la educación dual y podría afirmarse que ya existe una cultura de la educación dual, tanto en el ámbito educativo como en el empresarial, la cual se asume como una actividad necesaria para el desarrollo y la formación de personas. Además, el gobierno alemán contempla dentro de sus programas de cooperación internacional el aporte a países latinoamericanos que deseen incursionar en esta práctica educativa.

Es importante referirse a experiencias en Latinoamérica en la formación dual, la cual es diversa, pues varios países desarrollan propuestas en instituciones de educación técnica a nivel de secundaria, así como a nivel universitario, a partir de adaptaciones del sistema dual alemán, en este apartado se presenta una aproximación a las experiencias de Chile, México, Colombia y Costa Rica, aunque otros países latinoamericanos han incursionado también en ella.
La formación dual en Chile se desarrolla en educación técnica, en el nivel de secundaria cuenta con un programa en el sistema estatal, mediante una estrategia educacional en alternancia liceo y empresa, desde 1993, y tiene como propósito preparar a los jóvenes de tercer año de secundaria para su futuro desempeño laboral.

El programa chileno en formación dual tiene una duración de dos años, en el cual los aprendices se integran a procesos productivos en empresas, dos o tres días a la semana y el resto de días reciben formación académica en el liceo. Para el desarrollo de esta modalidad de aprendizaje (Sevilla y Balmaceda, 2001) indican que el estado asume los costos para cada alumno adscrito al programa, así también el liceo recibe una subvención estatal, no se consideran remuneraciones salariales para los aprendices. Las empresas brindan experiencia laboral en concordancia con lo aprendido en el liceo, mediante un proceso de instrucción adaptado a la realidad de cada empresa y bajo la dirección de un trabajador capacitado para ese rol maestro-guía.

Según (Sevilla y Balmaceda, 2001) en el año 1999 la educación dual chilena contaba con 5.999 alumnos aprendices que alternaban su formación en 78 liceos y 2.273 empresas adscritas al sistema. El $52 \%$ de los liceos y el $60 \%$ de estas empresas se concentraba en las Regiones Octava y Metropolitana.

México introduce la formación dual a partir de la necesidad de mejorar los procesos de formación del capital humano por la demanda de personal calificado. Algunas empresas que trabajan con el sistema de formación dual son las representantes de compañías como Volkswagen y Mercedes Benz, que implementan la modalidad según los manuales de la casa matriz en Alemania, mientras que otras sí lo hacen, buscando adaptaciones a la situación local.

La formación dual en México está relacionada con empresas automotrices, químicas, eléctricas y electrónicas, de alto nivel tecnológico. La mayoría de empresas 
que se comprometen con ella son alemanas. La procedencia alemana, según (Labarca, 1998), es reconocida por las empresas; incluso algunas de ellas, como Siemens, enfatizan ese aspecto considerándolo parte de la cultura de la empresa.

La experiencia de Colombia en formación dual data del año 1975, a partir del apoyo que el gobierno alemán brindó a ese país mediante un proyecto con universidades y cámaras de comercio con el propósito de formar personas con conocimientos sobre la realidad económica del país.

En Colombia también la educación dual ha sido adaptada del sistema alemán en el que involucra empresas públicas y privadas y está orientado a la formación técnica, parauniversitaria y universitaria, mediante un trabajo basado en proyectos previamente concertados entre la empresa y la institución educativa. Ese país se orienta hacia la extensión de la educación dual, en la actualidad las universidades Uniempresarial, la Coorporación Universitaria Empresarial Alexander Von Humboldt de Armenia y las universidades Autónoma de Occidente (UAO), la Tecnológica de Bolívar (UTB) y la Autónoma de Bucaramanga (Unab) desarrollan programas en esta modalidad de aprendizaje.

La modalidad dual en Costa Rica, tal y como indica (Mittmann, 2001) es conocida como el "Sistema Alemán", al igual que las demás experiencias latinoamericanas, corresponde a una adaptación de ese país; por tanto, se entiende por modalidad dual una formación en dos lugares de aprendizaje: un centro de enseñanza y una empresa, similar al sistema desarrollado en Alemania, ya que ha sido una adaptación de acuerdo con las condiciones socioeconómicas de las instituciones públicas costarricenses.

En Costa Rica, la educación dual concebida como un proceso sistemático ha tenido una aplicación reciente, la primera experiencia es del Instituto Nacional de Aprendizaje (INA) en el año 1993, en que se lleva a cabo el primer proyecto de aprendizaje bajo la modalidad dual y que se encuentra vigente en programas de formación como los de Mecánica Automotriz y Diseño Gráfico. De esta forma el técnico graduado en esas especialidades se gradúan con un valor agregado, la experiencia dual.

Otra experiencia, la del Ministerio de Educación Pública de Costa Rica (MEP) según (Rojas, 1999) fue la del Colegio Vocacional Monseñor Sanabria, en el año 1995, donde se desarrolló el Proyecto Dual en la carrera técnica de Mecánica Automotriz, actividad coordinada con el INA, y la Asociación de Propietarios de Talleres de Mantenimiento Industrial (APTAMAI), con la base curricular del programa de Mecánica Automotriz, aprobado por el Consejo Superior de Educación para el grado de Técnico Medio, cuyo programa se adaptó a la educación dual. Además en esa misma institución existe una propuesta para la Implementación del Sistema Dual en la Especialidad de Secretariado, que propone el Sistema Dual como un logro y una alternativa para definir el tipo de estudiante que requiere la sociedad costarricense, y que es formado en un proceso productivo que le permita enfrentarse al entorno laboral, logrando ampliar conocimientos y obtener mayor experiencia.

La experiencia del MEP, como lo indica (Mittmann, 2001) se fortaleció con los lazos de cooperación entre el Instituto Nacional de Aprendizaje y el Ministerio de Educación Pública, así como del apoyo que recibió el proyecto piloto en Educación Dual del Colegio Técnico de OsterholzScharmbeck en Alemania, a partir de un convenio de amistad y cooperación como parte del programa del Ministerio de Educación del Estado Federado de Baja Sajonia y de la Fundación Alemana para el Desarrollo (DSE), lo que significó tierra fértil para el desarrollo de esta nueva modalidad de aprendizaje, ya que mediante intercambio entre ambos países se logró capacitar a profesores. Así se implementó la formación dual en una institución educativa técnica en Costa Rica, adaptando el sistema de Educación Dual de Alemania, sobre 
la base del enlace entre la educación formal y el sector productivo del país.

Otra de las instituciones educativas costarricenses que ha incursionado en la formación dual es la Universidad Nacional, mediante el Proyecto de Extensión 010342 que desarrolla la Escuela de Secretariado Profesional desde el año 2000, con el afán de cumplir con los fines y objetivos de la Universidad Nacional y su compromiso con el desarrollo integral de los miembros de la comunidad universitaria, basado en el estudio de la realidad nacional.

La experiencia en la Escuela de Secretariado Profesional se lleva a cabo mediante el intercambio de colaboración con el Instituto Nacional de Aprendizaje, institución pionera en Costa Rica en formación dual. De esta manera se logra introducir, por primera vez, esta modalidad educativa en el nivel universitario, experiencia que durante los últimos cinco años ha dado como resultado la formación de profesionales en Administración de Oficinas, quienes se gradúan con experiencia laboral debidamente certificada (Universidad Nacional, 2005-2008).

Las relaciones de coordinación para ejecutar la formación dual, las realiza esa Unidad Académica mediante enlaces de cooperación con empresas públicas y privadas, que asumen estudiantes del Proyecto; además, esta Unidad Académica proyecta realizar enlaces cooperativos con las diferentes cámaras comerciales, industriales, turísticas y otras del país, para brindarle al estudiante diversidad de opciones empresariales.

Estas primeras experiencias en Costa Rica se fundamentan con el respaldo de las autoridades educativas. El 12 de abril de 2000, el Presidente de la República y los Ministros de la Presidencia, de Planificación y Política Económica, de Educación Pública y de Ciencia y Tecnología decretan "La creación e integración de la Comisión Nacional de Fomento de la Educación Dual (CONAFODUAL)" (Decreto No. 29079MEP), con miras a establecer un órgano de enlace entre los proyectos de desarrollo de esta modalidad y el Sistema Integrado Nacional de Educación Técnica para la Competitividad (SINETEC), creado también por Decreto Ejecutivo No. 27113-MPPLAN en 1998.

En relación con las necesidades de legislación laboral para la formación dual (Oviedo y Vargas, 2003) en su estudio sobre la perspectiva del derecho comparado en la relación laboral y la formación dual, entre sus conclusiones plantean la necesidad de adaptar formalmente una legislación costarricense que permita la articulación institucional, específicamente recomiendan un instrumento jurídico, como es un contrato especial fundamentado en un reglamento que establezca un equilibrio entre los intereses de la empresa, del estado o entidad educativa y de los aprendices.

A pesar de la carencia jurídica que facilite la aplicación de la formación dual en condiciones óptimas en los enlaces de cooperación entre las entidades participantes y el estudiante, la experiencia de INA, del Colegio Técnico Vocacional Monseñor Sanabria y de la Universidad Nacional ha representado un aporte sustantivo en términos de valor agregado a la formación técnica y profesional, un insumo significativo para la planificación curricular en las especialidades desarrolladas en esta modalidad, así como para orientar el perfil del egresado según los requerimientos de la empresa. Para la empresa ha sido un aporte en términos de transferencia de conocimientos.

En ese sentido es necesario considerar que a partir de esta modalidad de enseñanza y de aprendizaje es factible el desarrollo del potencial productivo del estudiante, lo que hace pertinente este modelo pedagógico, tanto en términos de efectividad y aporte a la formación del capital humano en un proceso activo y comprometido como del aprovechamiento de los recursos que tienen las empresas para sustituir la carencia de recursos de los sistemas educativos públicos. De esa 
manera se confirmaría la relación experiencia-educación como principio básico de la modalidad dual y que se explicita en el siguiente capítulo.

\section{Relación educación-trabajo}

La educación, en su mayoría de experiencias, ha representado un sistema formal orientado a la consecución de un título al final de una carrera que acredite al profesional para desempeñarse en un puesto de trabajo, a esto se le denomina educación tradicional; sin embargo, las nuevas exigencias del mercado en términos de aptitudes laborales han obligado a los sistemas educativos a complementar esa formación académica para establecer una estrecha relación entre la educación académica y la experiencia, producto de la necesidad de profesionalización para responder a las exigencias de los nuevos sistemas productivos.

En el proceso de formación intervienen tres figuras imprescindibles el estudiante, la empresa y la entidad educativa. El estudiante como actor principal, hacia su formación es que se orientan todas las acciones de un proyecto educativo en esta modalidad. La empresa como entidad formadora a partir de la actividad didácticoproductiva, que por medio del instructor o tutor lleva a cabo la enseñanza en un puesto de trabajo, ese tutor o tutores asumen un papel de maestros en la práctica. La institución educativa, representada por profesores, quienes tienen la responsabilidad de orientar o guiar la enseñanza académica en coordinación con el resto de docentes de la institución educativa.

Al considerar esos sujetos interactuando en un proceso de enseñanza la educación dual se concreta en esa relación aprendizaje teórico en el aula alternado con el aprendizaje en la práctica en un puesto de trabajo.

La formación dual como actividad de integración social y de desarrollo personal se convierte en factores que se complementan para potenciar las capacidades de los estudiantes para que respondan a las necesidades empresariales. Ese dinamismo pedagógico necesario para desarrollar esas capacidades las expresó (Dewey, 1960) quien apela a la experiencia, al aprendizaje con libertad y expresión para superar la imposición de programas que cultivan la individualidad y el aprendizaje rígido de un contenido, sobre lo que manifiesta que el aprender de textos y maestros, el aprender mediante la experiencia y a la adquisición de destrezas y técnicas aisladas se opone a la mínima utilización de las oportunidades.

Esa propuesta de combinación educación-trabajo fundamenta la educación dual como la forma de relacionar a los alumnos en nuevos campos, en los que deben aplicar los conocimientos, seleccionar y disponer las condiciones que influyen en su experiencia presente, así también la institución educativa debe brindar una posibilidad de experiencia creciente, como lo mencionaba (Dewey, 1960) el sistema de educación debe utilizar del método científico en el desarrollo de las posibilidades de una experiencia progresiva. Ese principio asienta la educación con una visión hacia el individuo y su desarrollo en y para la sociedad.

La concepción de la educación para el trabajo, según (Silveira, 1998), exige tres niveles de integralidad: la utilidad, el conocimiento técnico y las aptitudes, y las condiciones, medios, recursos y estrategias. De esta manera, la formación dual fundamentada en la educación para el trabajo basa su éxito en la ventaja del trabajo individual y colectivo de manera que articule la realidad con los requerimientos del desarrollo económico, la equidad social y la promoción de todos los grupos o sectores que se benefician o interactúan.

Por esa razón los sistemas productivos de mercado y tecnológicos, con miras a la eficiencia y la calidad, aseguran en forma simultánea las competencias necesarias del recurso humano al considerar el 
conocimiento técnico y las aptitudes sociales en forma equilibrada. La persona puede relacionar el saber con la ejecución y las condiciones, medios, recursos y estrategias en estrecha relación con el desarrollo personal, formación profesional, sector productivo y comunidad, que se integran por medio de políticas para facilitar su desarrollo.

El nivel de integración del conocimiento teórico y la práctica es la que establece el principio de la formación dual y que se estructura en la capacitación del capital humano que requiere la empresa y que está estrechamente vinculada con la demanda de personal calificado, cuyas necesidades son diversas y complejas en las diferentes áreas productivas. Mediante esa educación en alternancia, en forma simultánea en establecimientos escolares y en condiciones reales de trabajo, se organizan los sistemas de relaciones entre el proceso productivo y la capacitación. Esa relación también se fundamenta en combinar las competencias transmitidas en la institución formal y las adquiridas en la práctica profesional para beneficiar a ambas y fortalecer la igualdad de oportunidades ante el empleo, en ese sentido el Libro Blanco (1995), en la comunidad europea, establece entre sus objetivos la prioridad de acercar la educación al mundo del trabajo.

A partir de esa concepción, la integración social y el desarrollo personal orientado hacia la empleabilidad, las necesidades individuales y las del sector productivo y por ende la economía de un país, así como la utilidad de la educación para el trabajo individual y colectivo con miras a la eficiencia y la calidad, es que se aseguran en forma simultánea las competencias necesarias del recurso humano.

En los factores que orientan el principio de la formación dual, educación-trabajo, se establecen los fundamentos curriculares en que se debe cimentar un programa curricular en esta modalidad, que se detallan más adelante y que debidamente aplicados favorecen la formación profesional del estudiante.
Por tanto, la educación dual está caracterizada por el currículo como proceso tecnológico y con enfoque humanista, por su orientación hacia el educando como un ser interactivo y adaptativo, de quien se espera un papel activo en la resolución de problemas o situaciones reales del medio. También del docente se espera un papel de orientador o guía del proceso por medio de la articulación interdisciplinaria.

El currículo humanista fundamenta la educación dual en sus procesos pedagógicos que involucran el aprender a ser en el desarrollo de habilidades personales como la seguridad en sí mismo, la autoestima, la responsabilidad individual, la autonomía, la sociabilidad y el sentido de propósito; las habilidades interpersonales y sociales, los valores, el trabajo en grupo, la relación interpersonal, la capacidad de negociación, el saber escuchar y comunicarse y el manejo de la diversidad, todas exigencias en la actividad de interacción empresa-academia, las relaciones con otros y las actividades individuales.

La formación dual, desde el punto de vista del currículo como tecnología, según (Castillo, 2003), involucra la búsqueda y el uso de los medios para conocer y aprender, especialmente en la actividad propia del mundo laboral, por la capacidad de trasladar a la práctica el acervo teórico y el cultivo de una actitud científica, de habilidades tecnológicas y técnicas relacionadas con un oficio, una profesión o una especialidad que se denomina actividad didáctica-productiva, base de esta modalidad educativa o el aprender a hacer.

Considerando la complementariedad de los enfoques tecnológico y humanista es que la formación dual se plantea desde perspectivas curriculares que instituyen la formación dual como modalidad de enseñanza y de aprendizaje, cuyo principio fundamental es la relación educación-trabajo por medio de un enlace cooperativo entre la institución educativa y la empresa, con miras a formar un ciudadano altamente comprometido con la filosofía del país en 
que se desarrolla. En el caso de Costa Rica, con principios de libertad, solidaridad y de convivencia en paz con las diferentes culturas que interactúan, por lo tanto, la formación profesional en esta modalidad forma una persona académicamente, de acuerdo con las necesidades de los procesos productivos emergentes, y con valores humanos que exaltan el más noble espíritu del ciudadano costarricense.

\section{Fundamentos curriculares de la Formación Dual}

El currículo, entendido como el curso de la enseñanza y el aprendizaje sistemáticamente organizado en sus diferentes niveles macro, meso o micro, está basado en un proyecto curricular que pretende explicitar las intenciones de un sistema educativo y orientar la práctica pedagógica para responder a las grandes interrogantes de la educación ¿por qué? ¿para qué? ¿cómo? ¿a quién? ¿cuándo? ¿con qué enseñar? y ¿qué y cómo evaluar?

En la formación dual esas preguntas tienen respuesta en los diferentes factores que conducen a una concepción de ser humano que debe responder a los requerimientos de formación para desempeñar un puesto de trabajo con eficiencia y eficacia, según las necesidades de competitividad en un mundo globalizado, en el que los sistemas productivos deben atender a mercados cada vez más amplios y competitivos, asimismo debe poseer cualidades para la convivencia en paz y solidaridad; por tanto, la formación dual se desarrolla desde un enfoque tecnológico y humanista.

El fundamento filosófico de la formación dual se establece en el desarrollo de un proceso en el que intervienen estudiantes, profesores, instructores de empresa o tutores y otros funcionarios por medio de la interrelación en el proceso productivo. Esa interrelación de los sujetos participantes exige en el diseño curricular una orientación con principios y valores que expresan el modelo de ser humano y de sociedad que se pretende formar, considera así la ideología de un país, los criterios de libertad y equidad que orientan los fines educativos, por tanto al plantear un proyecto de esta naturaleza se deben considerar los propósitos educativos que prevalecen y responder a la pregunta ¿para qué educar?

Los principios filosóficos mayores están plasmados en los fines o propósitos de la educación y que guían la formación con bases ideológicas en las que prevalece la integración de desarrollar aptitudes, atendiendo adecuadamente las diferencias individuales.

Desde esa perspectiva, la capacidad productora tiene una estrecha relación con los intereses del individuo, establecido en las políticas y estrategias educativas del contexto en que se desarrolle, para el logro de la unidad de la educación y el trabajo, a partir de la aplicación en un proceso educativo de los cuatro pilares de la educación, propuestos por (Delors, 1996) aprender a conocer, aprender a hacer, aprender a ser y aprender a vivir, que se detallan más adelante, y que constituyen orientaciones filosóficas de la formación dual.

El fundamento epistemológico como rama de la filosofía que trata de los problemas filosóficos que rodean la teoría del conocimiento, se ocupa de la definición del saber y de los conceptos relacionados, de las fuentes, de los criterios, de los tipos de conocimiento posible y del grado con el que cada uno resulta cierto; así como de la relación exacta entre el que conoce y el objeto conocido.

La formación dual considera diversos tipos de conocimiento y que cada uno de ellos se refleja en determinados propósitos, perspectivas, experiencias, valores e intereses. El modelo de James Banks, tal y como lo analiza (Torres, 1996), identifica varias modalidades de conocimiento a través de los cuales las personas construyen sus explicaciones o interpretaciones de la realidad. 
Además contempla el constructivismo orientando la propuesta hacia las corrientes educativas de: aprender a aprender, aprendizaje significativo, desenvolvimiento individual y grupal, y formación para la vida y el trabajo, que establecen la pedagogía de la formación en las actividades de interacción que desarrolla el estudiante en el aula y en un puesto de trabajo.

La formación dual establece el conocimiento en el análisis e intervención de la realidad a partir de la experiencia humana, de la identificación de problemas en la realidad laboral, y en el aula por medio de una organización disciplinar que involucre el contexto del estudiante como ente generador del significado del conocimiento, que comprende la potenciación de ideales personales, sociales y profesionales de un trabajador que le garanticen el éxito laboral, considerando que las empresas actuales exigen un alto rendimiento y efectividad en los procesos productivos y de servicios que se ha convertido en un imperativo de supervivencia en mercados globales o mundiales, así el desarrollo de su personal y sus métodos de trabajo orientan el quehacer de empresas emprendedoras, competitivas y por ende triunfadoras.

El Fundamento psicopedagógico en la formación dual se desarrolla a partir de la interacción academia-empresa-alumno, en el que se enriquecen todos, la entidad educativa a partir de la sistematización de experiencias que le permite integrar la educación para el trabajo considerando la utilidad, el conocimiento y aptitudes, las condiciones materiales y el contexto. Por su parte la empresa por medio de la formación corporativa recibe un aporte del estudiante en cuanto a nuevos conocimientos teóricos, mediante la interacción con el Instructor de Empresa o Tutor y que a su vez le permite la mejora en los procesos.

Las fuentes psicopedagógicas en la formación dual comprenden aporte de la psicología y de la pedagogía que orienta el proceso de enseñanza y de aprendizaje en dos lugares distintos la empresa y la academia, en ambos se consideran los principios de autonomía del individuo, el equilibrio entre la adquisición de conocimientos y las competencias en procesos colaborativos, a partir de las necesidades individuales, empresariales y sociales y el énfasis en los procesos más que en los resultados.

Considerando lo anterior, la orientación pedagógica es flexible y dinámica en un proceso de desarrollo de la persona en interacción con la sociedad, basada en la efectividad de la relación educaciónexperiencia, en un proceso interactivo de los sujetos que participan: el estudiante, la institución educativa con figuras del docente como facilitador en el aula y en la etapa de seguimiento en la empresa, y el o los funcionarios o tutor (es) que aporta la empresa, así como las condiciones materiales que ofrecen esas dos instituciones, a partir de una concepción curricular centrada en la persona y la concepción curricular participativa social.

La formación dual requiere un currículo integrador que permita concebir los contenidos a partir del aprendizaje cooperativo de temas, problemas o proyectos concretos que usualmente surgen del análisis de necesidades de la realidad, prevalece la interdisciplinariedad y una estrecha relación teoríapráctica, con bases de autonomía, principios éticos y de solidaridad, un espíritu crítico y conciencia colectiva. Busca el desarrollo de la persona para potenciar sus capacidades y las del grupo que lo rodea, de esta manera logra un cambio social en términos de desarrollo humano en comunidad y con respeto al equilibrio de la naturaleza, fundamentos básicos de la educación dual.

La formación dual, desde la perspectiva psicopedagógica también se basa en los cuatro pilares fundamentales de la educación, según la UNESCO: Aprender a conocer, aprender a hacer, aprender a vivir, aprender a ser, a partir de una concepción de la educación en la que se asume el educando como protagonista de la práctica profesional y formado de manera integrada sustentada en una formación técnico-humanista. 
En la formación dual el aprender a conocer supone aprender a aprender, ejercitar la atención, la memoria y el pensamiento que le permitan articular entre lo concreto y lo abstracto. El aprender a hacer está relacionado con la formación profesional, no solo aprender un oficio o una profesión, en un sentido más amplio involucra la adquisición de competencias que le permitan afrontar situaciones que faciliten la formación técnica y profesional, el comportamiento social, la aptitud para trabajar en equipo, con iniciativa y decisión para asumir riesgos. Esas aptitudes involucran una estructuración de temas o contenidos curriculares que enseñen esas cualidades en los estudiantes.

La relación directa entre el pilar aprender a hacer como adquisición de competencias laborales (Tünnermann, 1997) lo relaciona indicando que las competencias y las cualidades pueden adquirirse más fácilmente, si los alumnos y estudiantes tienen la posibilidad de ponerse a prueba y de enriquecer su experiencia participando en actividades profesionales y sociales, al tiempo que cursan sus estudios. Eso justifica la importancia, cada vez mayor, que debe dársele a las diversas formas posibles de alternancia entre la escuela y el trabajo.

$\mathrm{El}$ aprender a vivir contempla la convivencia con los demás y el aprendizaje de los más altos valores humanos de convivencia social y el respeto de uno mismo y por la otredad, por medio de un sistema de conocimiento de los demás, de su historia, de sus tradiciones y su espiritualidad, la necesidad de interdependencia y los desafíos futuros en términos de diversidad cultural.

El principio aprender a ser aplica en la formación dual en términos de la necesidad de desarrollar la capacidad de autonomía, de tomar decisiones y de asumir con responsabilidad los resultados de las acciones y que en el desarrollo de sus conocimientos intelectuales, prácticos y de relaciones humanas se constituye en forma integral un ser humano con principios de libertad y buen juicio para la convivencia en sociedad.
Considerando esos principios, el diseño de un proyecto educativo en formación dual considera una concepción curricular que recoge las intenciones educativas que expresan los fines, principios y objetivos de la institución educativa. Esas intenciones curriculares se deben orientar al desarrollo del individuo con un papel activo en torno a la sociedad a la que pertenece y a quienes debe responder.

En ese sentido la praxis como la visión idealista y humanista de vincular la reflexión y la acción con una orientación práctica considera la vida concreta y cotidiana como sinónimo de acción y como la situación social del individuo en el trabajo productivo, por tanto el individuo debe atender sus necesidades, sus intereses, sus problemas, su conducta y su personalidad integral. La sociedad como una totalidad estructural debe considerar las necesidades de cada elemento que la constituye como lo socioeconómico, lo sociopolítico y sociocultural, la naturaleza como ecosistema, su dimensión biológica y física y como visión ontológica del mundo.

La formación dual establece la orientación pedagógica hacia un aprendizaje visto como un sistema de componentes de carácter holístico, en términos de conocimientos conceptuales, de procedimientos organizados en esquemas operacionales y que permitan dentro de un grupo la identificación de tareas o problemas y su resolución de manera eficaz. Ese componente cognitivo lo plantea como un medio por el cual el ser humano conoce el mundo que le rodea y que influye de modo directo en la transformación de la realidad y con esto transforma al individuo por medio del proceso de metacognición para relacionar los conceptos con los procedimientos e incorporarlos como habilidades, la forma de pensar, las sensaciones y las percepciones ante la resolución de problemas reales. Ese proceso a lo interno del individuo lo relaciona por medio de la interacción de las motivaciones externas.

En la formación dual, a diferencia de la tradicional, el estudiante identifica los 
resultados esperados en el medio del trabajo para dominar la tarea, toma en cuenta los criterios de desempeño que implican responsabilidad de su propio aprendizaje. La diferencia entre ambas modalidades, la tradicional y la dual, está en que la segunda contextualiza en una situación real la meta que se desea lograr en términos de aprendizaje, así como la implicación del individuo en ese proceso metacognitivo, la tradicional lo hace a partir de situaciones simuladas.

En ese proceso de aprendizaje no solo intervienen los componentes cognitivos sino también las motivaciones, la metacognición, las cualidades y el comportamiento del individuo. Desde esta perspectiva la orientación del aprendizaje es propia del modelo centrado en el estudiante, ya que toma en cuenta sus propias necesidades de aprendizaje, su interés y sus motivaciones, así la potencialidad de desarrollo humano y profesional y que se relaciona con la complejidad de los caracteres, la complejidad del puesto de trabajo y la relación con otros. Además, toma en cuenta el enfoque personológico del proceso de educación técnica y profesional, ya que integra los componentes de la persona: el conocimiento, la motivación, la metacognición y las cualidades de la personalidad.

En esta modalidad la estructura didáctica respeta lo que un individuo necesita saber, hacer y ser, según las exigencias de la profesión para la que se está formando, potenciando su preparación para la vida. El papel del docente debe ser mediador del proceso de educación técnica y profesional creando situaciones de aprendizaje que ponga al estudiante en una posición crítica, reflexiva, participativa, con un papel protagónico, que reflexione y analice cómo aprende, qué le falta y cómo es como persona, a partir de la práctica por medio de la vivencia y experiencia del estudiante, el docente y el contexto real como base curricular, además se aprecia la constante y clara deliberación.

El contenido lo determina la base de la tarea y sus procedimientos. Los métodos de enseñanza, los medios y la forma de organizarlos están en función de una enseñanza y aprendizaje desarrolladores, el sujeto es protagonista y productivo por medio del principio pedagógico estudiotrabajo.

La formación dual tiene la perspectiva de desarrollar la capacidad productiva del individuo con sentido de eficiencia y efectividad social, por lo que los fundamentos socioeconómicos asientan el currículo en esta modalidad, con la visión de formación del recurso humano para la productividad. El componente socioeconómico está orientado al desarrollo económico y social porque está orientado a la relación aprendizajecompetitividad de las nuevas orientaciones mundiales del trabajo.

En esa orientación, la enseñanza es una condición imprescindible para la vida y para el trabajo, cuyo principio básico es el criterio social en la instrucción, encaminada hacia las necesidades de formación en las sociedades avanzadas, que se preocupan por la capacidad de los individuos, la búsqueda de la eficiencia y la competitividad. Hacia esas perspectivas de formación es que se vislumbra la educación con fines de capacitar ciudadanos hábiles para alcanzar los objetivos de aprender, de pensar, de solucionar problemas y de ser creativos en torno a las necesidades del mercado local y mundial.

Es así como los fundamentos curriculares identifican las bases sociales y culturales en los cuales debe estar cimentado un proyecto curricular orientado hacia la búsqueda de proceso de investigación, de análisis y generación de aportes para transformar la realidad.

Esa realidad comprende el entorno en que el estudiante se desenvuelve como ser individual y social, cuyas responsabilidades consigo mismo y con los demás trasciende la formación teórica para llevarla a la práctica y aprender en un puesto de trabajo.

Esa práctica se ubica en un sistema complejo como es la organización y que a 
partir del aprendizaje de las tareas puede dar un aporte sustantivo al quehacer, con mayor propiedad, ya que el proceso didáctico productivo en que está inmerso en la modalidad de formación dual, le permite la interacción con estados que lo obligan a pensar, analizar, tomar decisiones y actuar ante las situaciones cambiantes de la realidad empresarial.

Esa realidad empresarial a la que se somete el estudiante, está a su vez inmersa en un mundo de oportunidades, que lo ubican en un espacio y en un tiempo productivo, el cual está afectado por una estructura política, económica y social que debe sobrellevar para lograr la integralidad de su formación, por tanto un proyecto de formación dual debe orientar sus esfuerzos a la formación de las máximas expresiones del ser humano, que se deben plasmar en un diseño curricular y que están enmarcadas en los fundamentos curriculares para el logro de ciudadanos responsables, creativos, con conciencia de su responsabilidad de búsqueda de sostenibilidad ambiental, con valores y actitudes, capaces de crear, emprendedores y competentes.

Para ese logro del potencial humano y profesional es importante establecer condiciones de aprendizaje idóneas a las necesidades individuales de cada persona y a las exigencias tecnológicas y procedimentales de la empresa actual, manteniendo un equilibrio entre la calidad, el costo y la protección del medio ambiente, la interacción multicultural y las perspectivas de intercambio comercial y cultural con otras naciones.

Por tanto, las fuentes curriculares y su concepción de la educación se convierten en filtros para seleccionar y organizar objetivos, contenidos, actividades metodológicas, técnicas y recursos educativos con fines de reproducir la cultura de desarrollo por medio de la entrega docente y la actividad didáctico-productiva en una relación estrecha experiencia-educación pero asentada en principios humanistas.

\section{Referencias bibliográficas}

Caillods, F. (s. f). Las reformas de la educación secundaria en países de Europa. Extraído el 15 de marzo de 2007, de http://www.oei. org.ar/edumedia/pdfs/T05_Docu6 Lasreformasdelaeducacion_Caillods. $\underline{\mathrm{df}}$

Castillo, L. (2003). Enfoques o concepciones curriculares. Instituto Profesional de Providencia. Escuela de Educación. Educación Básica. [Resumen] (s.p.). Santiago, Chile. Extraído el3 de marzo de 2003 de

http://www.asesoríaspedagogicas.cl/ ipp/clase.curriculum.doc

Delors, J. (1996). La Educación Encierra un Tesoro. Madrid: Santillana/Ediciones UNESCO.

Dewey, J. (1960). Experiencia y Educación. Buenos Aires, Argentina: Ed. Losada.

Dewey, J. (1976). Democracia y Educación. Buenos Aires, Argentina: Ed. Losada.

Díaz, J., Márquez, J. y Meléndez, L. E. (2002, Setiembre-Diciembre). Estimación del potencial de recursos humanos bajo el enfoque de competencias. Revista Encuentro Educacional. Revista especializada en educación, 3(9), 358-371.

Escudero, J. M. (2000). Diseño, desarrollo e innovación del currículum. Madrid: Editorial Síntesis, S.A.

Gallart, M. A. y Jacinto, C. (1995). Competencias laborales: tema clave en la articulación educación-trabajo. Boletín de la Red Latinoamericana de Educación y Trabajo, CIID-CENEP, 2, Año 6, sin paginación. Extraído el 22, julio, de 2007 de http://www.oei. es/oeivirt/fp/cuad2a04.htm 
Hernández, P. (2000). Diseñar y enseñar. Madrid: Ed. Narcea. S.A.

Labarca, G. (1998). Formación para el trabajo en industrias mexicanas (Documento desarrollado en el marco del Proyecto "Políticas y estrategias para mejorar la calidad, eficiencia y relevancia del entrenamiento y de la educación técnica y profesional en América Latina y el Caribe"). Santiago de Chile: CEPAL-GTZ Extraído el 15 de marzo, de 2007 de http://www.cinterfor.org.uy/public/ spanish/region/ampro/cinterfor/ dbase/ret/ges_for/xii_index.htm

Libro Blanco. (1995). Libro Blanco sobre la educación y la formación. "Enseñar y aprender. Hacia la sociedad Cognitiva" Bruselas: Comisión de las Comunidades Europeas, sin paginación. Recuperado el 22 de noviembre de 2006 de la consulta de http://www.uhu.es/cine.educacion/ didactica/1libroblanco.htm

Ministerio de Educación Pública [MEP]. (s.f.). Ley Fundamental de Educación de 1957 (Ley No. 2160), Capítulo I. De los Fines de la Educación Costarricense. Extraído el 24 de noviembre de, 2006 de http://www. portal.unesco.org/education/en/ files/27617/10717533461Costa_Rica. doc/Costa+Rica.doc

Mittmann, F. (2001). Educación dual en Costa Rica: proyecto piloto mecánica automotriz del Colegio Vocacional Monseñor Sanabria 1996-1999. San José, Costa Rica: Instituto Nacional de Aprendizaje.

Neave, G. (2001). Educación Superior: Historia y Política. Estudios comparativos sobre la universidad contemporánea. Barcelona. España: Editorial Gedisa.
Oviedo, J. y Vargas, T. (2003). Relación Laboral y Formación Dual: Una Perspectiva del Derecho Comparado. Tesis de licenciatura para la obtención del título de Licenciado en Derecho. TBG 22291. Facultad de Derecho. Universidad de Costa Rica.

Rojas, A. (1999). Implementación del Sistema de formación dual en la especialidad de secretariado en el Colegio Vocacional Monseñor Sanabria. Práctica dirigida de licenciatura para la obtención del título de Licenciada en Ciencias de la Educación con énfasis en Administración Educativa.TFG 19687. Facultad de Educación. Escuela de Administración Educativa. Sede Regional del Atlántico. Universidad de Costa Rica.

Rolf, A. (1997). Preguntas y Respuestas Relacionadas al Sistema de Formación Profesional Alemán. Alemania Federal, Joachim Munich: Editado por Ministerio Federal de Educación, Ciencia, Investigación y Tecnología.

Sandí, M. (2004). Taller para Maestros de Oficinas, Formación Dual en Administración de Oficinas. Universidad Nacional: Escuela de Secretariado Profesional, s.p.

Sevilla,M.P.yBalmaceda,F.(2001,Diciembre). Invirtiendo en Entrenamiento General: el Programa de Formación Dual. Revista de Análisis Económico, 2(16), pp. 137-153. Extráido el 15 de marzo de 2007 de http://www.dialnet.unirioja.es/ servlet/articulo?codigo $=237702$

Silveira, S. (1998, 5 al 7 de agosto). $L a$ educación para el trabajo: un nuevo paradigma. Trabajo presentado en el Seminario realizado en el Centro Internacional de Investigación y Desarrollo sobre Formación Profesional. Centro Internacional 
de Investigación y Desarrollo sobre Formación Profesional[CINTERFOR/ OIT], Panamá.

Stoner, J. y Wankel, Ch. (1989). Administración. (3era. ed.). México: Editorial Prentice Hall.

Tippelt, R. y Amorós, A. (2000). Manual didáctico: El Sistema Dual de Formación Profesionalen la República Federal de Alemania. Lima, Perú: Universidad Ludwing Maximiliam de Munich y Oficina de Cooperación Internacional (BIZ).

Torres, J. (1996). Globalización e interdisciplinariedad: el currículum integrado. Madrid. España. Ediciones Morata.
Tünnermann, C. (1997). La educación para el siglo XXI. Caracas, Venezuela: UNESCO.

Universidad Nacional Autónoma [UNA]. (2005-2008). Formación Profesional Dual en Administración de Oficinas y Educción Comercial. (Proyecto de extensión, vigente de julio 2005diciembre 2008, código 10349. Heredia: Escuela de Secretariado Profesional.

Vega, L. (2005). Evaluación Programa en Administración de Oficinas 2000-2004. (Informe de evaluador externo). Heredia: Universidad Nacional, Escuela de Secretariado Profesional. 
